\title{
Protecting those who serve: are we doing enough to prevent tuberculosis in healthcare workers?
}

\author{
Jennifer Furin ${ }^{1}$ and Giovanni Sotgiu (10 ${ }^{2}$ \\ Affiliations: ${ }^{1}$ Harvard Medical School, Dept of Global Health and Social Medicine, Boston, MA, USA. ${ }^{2}$ Dept of \\ Medical, Surgical and Experimental Sciences, Clinical Epidemiology and Medical Statistics Unit, University of
} Sassari, Sassari, Italy.

Correspondence: Giovanni Sotgiu, University of Sassari Medical School, Dept of Medical, Surgical and Experimental Sciences, University of Sassari, Via Padre Manzella 4, Sassari 07100, Italy. E-mail: gsotgiuda uniss.it

@ERSpublications

Tuberculosis in healthcare workers can be prevented http://ow.ly/Xke330o6B0N

Cite this article as: Furin J, Sotgiu G. Protecting those who serve: are we doing enough to prevent tuberculosis in healthcare workers? Eur Respir J 2019; 53: 1900485 [https://doi.org/10.1183/13993003.004852019].

The continuous flow of scientific articles on high tuberculosis (TB) morbidity and mortality over the past decades highlight a partial or total failure of national and local policies to address a potentially curable and preventable infectious disease. The ongoing transmission of Mycobacterium tuberculosis strains in the community and in healthcare settings proves the inability of the system to epidemiologically reverse the natural history of this ancient disease. Ironically, these hospitals and health centres, places where people go for healing, are places of high risk for $\mathrm{TB}$ infection and disease for those attending inpatient and outpatient settings, including for healthcare workers.

On the website of "TB Proof", a noted advocacy group made up of healthcare workers who have survived TB, Dr Heena Narotam writes "imagine being taught how to treat and prevent TB and, then, to step into the hospital environment to find that many of the preventive strategies are not being implemented" [1]. One needs only scroll through the page's testimonies from men and women who have become infected with $M$. tuberculosis and sick from TB while providing medical care, to see the upsetting consequences of such public health laxity in practice. Tragically, this suffering was unnecessary and unsolicited, and could have been avoided if basic TB prevention policies and practices had been implemented [2].

M. tuberculosis-related infection and disease among people working in healthcare settings is a pressing issue whose existence calls into question how seriously and systematically we can take the global commitment to "End TB" everywhere [3]. Studies dating back to the 1950s have shown that healthcare professionals have nearly double the rate of $M$. tuberculosis infection compared with those who do not provide healthcare services [4]. This is especially notable among people working in low- and middle-income countries (LMICs) where the TB burden is high in the community and in healthcare settings [5]. In spite of this fact, more stringent infection control practices to prevent nosocomial TB transmission seem "reserved" for healthcare settings in low TB burden countries, another example of double standards being practised in the field of TB [6]. Examples of failed administrative and/or environmental interventions have been reported [7] and have been attributed to the low priority given to this clinical and public health threat. 
Thus, it is alarming that the systematic review on the prevalence and incidence of $M$. tuberculosis infection among various cadres of workers in the healthcare setting, including students, reported by APRIANI et al. [8] in this issue of the European Respiratory Journal continues to document exceptionally high rates in these population groups. Their research analysed 85 studies from a heterogeneous group of LMICs using tuberculin skin testing (TST), interferon gamma-releasing assays (IGRAs) or both to document both the prevalence and the incidence of $M$. tuberculosis infection among persons working within healthcare settings. In their definition of healthcare workers, the authors included not only physicians, nurses and counsellors, but also cleaners, drivers and housekeepers. Although they found a significant heterogeneity, clear and worrisome trends emerged. Using TST and IGRAs a mean of 49\% and 39\% of healthcare workers were found to have prevalent $M$. tuberculosis infection, respectively. Using the same TST and IGRAs they found a mean of $17 \%$ and $18 \%$ of healthcare workers who developed incident $M$. tuberculosis infection, respectively. Lower percentages were seen in students and risk factors included the number of years working in the healthcare environment, the location of work, contact with a patient or co-worker who had TB, and engaging in counselling with, or sputum collection from, a person with TB.

The study was limited in that an individual patient data meta-analysis could not be performed and it did not assess information on TB disease. However, one notable finding of the research was that only 15 studies reported on TB infection control measures: of these none reported a full package of infection control measures and nine (60\%) reported inadequate (and/or inappropriate) implementation of existing TB infection control policies. Poor implementation of infection control practices occurs even though some of these policies should be straightforward to implement, including patient triage, improved air and patient flow. Provision of fitted personal protective devices should be as essential as the provision of gloves and these should be made available for all persons working in the healthcare system [9], including those typically overlooked in basic infection control programmes and activities that focus on training and education, including people who work as drivers, cleaners, transporters and housekeepers.

The most effective way to reduce M. tuberculosis transmission to healthcare workers is early diagnosis of infectious TB patients and initiation of effective and tailored anti-TB therapy [10]. A strategy known as "FAST" (Finding patients Actively, Safely separating them from others, and Treating them with effective therapy) has been shown to be effective in finding persons with TB who may be missed through usual passive screening methods and whose misdiagnosis may contribute to increased nosocomial M. tuberculosis transmission to healthcare workers, patients and visitors [11]. The effectiveness of this strategy, however, is hampered with the continued use of outdated (i.e. smear microscopy) and lengthy (i.e. solid culture) tools that cannot detect drug resistance, and ongoing prescription of ineffective drugs and regimens that fail to cure a substantial proportion of people living with the disease [12]. Patient and healthcare delays need to be addressed by improving the awareness and knowledge of TB risk in the general population and training healthcare workers on infection control policies and practices. More attention should be paid to risk assessment in healthcare settings, to the surveillance of epidemiological data on at-risk population groups, and to the implementation and scale-up of administrative and environmental measures, as well as distribution of personal protective equipment.

One important aspect of TB prevention is treatment of infection before it develops into active disease (i.e. preventive therapy for latent TB) [13]. Although this effective strategy is not mentioned in the paper, it has been shown to reduce TB disease risk among persons who have been exposed to contagious cases in the healthcare setting [14]. Increased efforts to improve access to and follow-up of treatment for M. tuberculosis infection are essential to reduce morbidity (and mortality) among healthcare workers, especially those with incident recent infection. This group should be a priority population when it comes to broader implementation of treatment of $M$. tuberculosis infection, and novel, shorter, effective and safe regimens (including 12-week and 1-month regimens of isoniazid and rifapentine) should be offered to all healthcare workers.

Finally, a free and confidential occupational health service should be offered as an essential component of infection control, not just for TB diagnosis and prevention but also to provide psychosocial support to people who are exposed to M. tuberculosis sources in the workplace [15]. Given the significant morbidity and mortality associated with $\mathrm{TB}$, as well as the discrimination and shame associated with the disease, people working in the healthcare field may need long-term counselling and support if they are found to be infected with $M$. tuberculosis.

TB has long been managed using a "one size fits all" approach, whose focus on simplicity may be one of the reasons limited progress has been made in the fight against the disease [16]. Using this strategy has meant that healthcare workers in resource-limited settings, and most notably those with high burden of $\mathrm{TB}$ disease, have not been given the attention and resources they merit to keep them protected from TB infection and disease. Embracing a human rights-based approach to TB would benefit these vulnerable 
individuals and allow them to remain champions in the fight to end TB. Everyone affected by TB deserves the best care possible but looking at how we consider and treat those who serve on the front lines shows we have a long way to go if we are to achieve TB elimination. Given the shortage of healthcare workers, particularly in the field of TB, it is time we stop offering them the bare minimum and instead insist on implementing for them the highest standard of TB prevention possible.

Conflict of interest: None declared.

\section{References}

TB Proof. Who We Are. www.tbproof.org/who-we-are/our-team/ Date last accessed: March 3, 2019.

2 World Health Organization. WHO Policy on TB Infection Control in Health-care Facilities, Congregate Settings and Households. Geneva, WHO Press, 2009. Available from: www.who.int/tb/publications/tb-facilities-policy/en/

3 World Health Organization. The End TB Strategy: Global Strategy and Targets for Tuberculosis Prevention, Care and Control after 2015. Geneva, World Health Organization, 2014.

4 Fennelly KP, Iseman MD. Health care workers and tuberculosis: the battle of a century. Int J Tuberc Lung Dis 1999; 3: 363-364.

5 Joshi R, Reingold AL, Menzies D, et al. Tuberculosis among health-care workers in low- and middle-income countries: a systematic review. PLoS Med 2006; 3: e494.

6 Shakow A, Admay C, Nicholson T, et al. Double standards in global health: medicine, human rights law and multidrug-resistant TB treatment policy. Health Hum Rights 2016; 18: 85-101.

7 Sotgiu G, D'Ambrosio L, Centis R, et al. TB and M/XDR-TB infection control in European TB reference centres: the Achilles' heel? Eur Respir J 2011; 38: 1221-1223.

8 Apriani L, McAllister S, Sharples K, et al. Latent tuberculosis infection in healthcare workers in low- and middle-income countries: an updated systematic review. Eur Respir J 2019; 53: 1801789.

9 Claasens MM, van Schalkywk C, du Tolt E, et al. Tuberculosis in health care workers and infection control measures at primary health care facilities in South Africa. PLoS One 2013; 8: e76272.

10 Van Custem G, Isaakidis P, Farley J, et al. Infection control for drug-resistant tuberculosis: early diagnosis and treatment is key. Clin Infect Dis 2016; 62: Suppl. 3, S238-S243.

11 Nathavitharana RR, Daru P, Barrera AE, et al. FAST implementation in Bangladesh: high frequency of unsuspected tuberculosis justifies challenges of scale-up. Int J Tuberc Lung Dis 2017; 21: 1020-1025.

12 Le H, Nguyen N, Tran P, et al. Process measure of FAST tuberculosis infection control demonstrates delay in likely effective treatment. Int J Tuberc Lung Dis 2019; 23: 140-146.

13 Reid A, Grant AD, White RG, et al. Accelerating progress towards tuberculosis elimination: the need for combination treatment and prevention. Int J Tuberc Lung Dis 2015; 19: 5-9.

14 Behrman AJ, Shofer FS. Tuberculosis exposure and control in an urban emergency department. Ann Emerg Med 1998; 31: 370-375.

15 Buregyeya E, Nuwaha F, Wanyenze RK, et al. Utilization of HIV and tuberculosis services by health care workers in Uganda: implications for occupational health policies and implementation. PLoS ONE 2012; 7: e46069.

16 Acquah R, Furin J. Universal regimens or universal access to drug susceptibility testing for tuberculosis? Lancet Infect Dis 2019; 19: 224-225. 